\title{
Cutaneous lupus concerns from the patient perspective: a qualitative study
}

\author{
Daisy Yan (D) , , Danielle Zamalin, ${ }^{1,2}$ Srita Chakka, ${ }^{1,2}$ Rebecca Krain, ${ }^{1,2}$ \\ Josef Concha, ${ }^{1,2}$ Rui Feng, ${ }^{3}$ Sarah Ahmed, ${ }^{1,2}$ Joyce Okawa, ${ }^{1,2}$ Victoria P Werth ${ }^{1,2}$
}

To cite: Yan D, Zamalin D, Chakka S, et al. Cutaneous lupus concerns from the patient perspective: a qualitative study. Lupus Science \& Medicine 2021;8:e000444. doi:10.1136/ lupus-2020-000444

DY and DZ are joint first authors.

Received 9 September 2020 Accepted 4 February 2021

Check for updates

(C) Author(s) (or their employer(s)) 2021. Re-use permitted under CC BY-NC. No commercial re-use. See rights and permissions. Published by BMJ.

${ }^{1}$ Dermatology, University of Pennsylvania, Philadelphia, Pennsylvania, USA ${ }^{2}$ Dermatology, Corporal Michael J Crescenz VA Medical Center, Philadelphia, Pennsylvania, USA ${ }^{3}$ Biostatistics, University of Pennsylvania, Philadelphia, Pennsylvania, USA

Correspondence to

Dr Victoria P Werth; werth@mail. med.upenn.edu

\section{ABSTRACT}

Objective There is a need to identify concerns unique to patients with cutaneous lupus erythematosus (CLE), which may not be captured by current common-practice dermatological quality-of-life tools. This study formally characterises what bothers patients with CLE about their disease by conducting semistructured, qualitative interviews.

Methods Sixteen patients with CLE were interviewed about how their cutaneous findings impact their daily life. Each interview was transcribed, coded and categorised for recurrent themes. Current CLE activity and damage were also assessed by the Cutaneous Lupus Activity and Severity Index tool.

Results Responses were categorised into six themes, including Fear of Disease Progression, Unwanted Attention, Self-Consciousness, Physical Signs/Symptoms, Emotional Symptoms and Functional Decline. The most commonly reported themes were Self-Consciousness, mentioned by 13 of $16(81.3 \%)$ patients, Physical Symptoms, mentioned by 12 of $16(75 \%)$, and then Fear of Disease Progression, by 11 of $16(68.8 \%)$. Frequently mentioned physical signs/ symptoms included erythema, itch, dyspigmentation, scar and alopecia. The physical signs/symptoms were categorised as activity signs/symptoms, damage signs and other. For activity signs, erythema was mentioned most frequently (5 of 16), then scale (2 of 16). For activity symptoms, itch was mentioned most frequently (6 of 16), then pain (5 of 16). For damage signs, dyspigmentation was mentioned most frequently (4 of 16), followed by scarring (3 of 16). Patients less than 60 years old were more likely to report emotional symptoms than older patients $(p<0.05)$, but there was no significant variation in frequency of reported themes between race, sex or subtype of CLE.

Conclusions These patient experiences and resultant themes elucidated by this study are worth noting in future standardised estimations of the quality of life of patients with CLE. Additionally, the concerns shown by these interviews are important topics for providers to discuss when evaluating patient disease progression.

\section{INTRODUCTION}

Lupus erythematosus (LE) is an autoimmune condition that can target multiple organ systems, and thus can either present as cutaneous LE (CLE) and/or SLE. A diagnosis of CLE can be further categorised into one of three main subtypes: chronic CLE, subacute

\section{Key messages}

What is already known about this subject?

- Ogunsanya et al, using a focus group approach, were among the first to capture cutaneous lupus patientreported outcomes with regard to quality of life.

What does this study add?

- Concerns unique to patients with cutaneous lupus erythematosus (CLE) are not fully captured by common-practice dermatology quality-of-life tools. This study aims to formally characterise what bothers patients with CLE about their disease by conducting semistructured, qualitative interviews. These conversations were conducted one-on-one in clinic rooms with the hope to provide an added sense of privacy and security, as compared with Ogunsanya's focus group approach.

- Themes were also grouped by patient characteristics such as race and sex to determine recurrent patterns.

How might this impact on clinical practice or future developments?

- The patient experiences and resultant themes elucidated by this study are worth noting in future standardised estimations of the quality of life of patients with CLE. Additionally, the concerns shown by these interviews are important topics for providers to discuss when evaluating patient disease progression.

CLE (SCLE) and acute CLE. ${ }^{1}$ Chronic CLE itself can be further subtyped into more specific forms, the most common of which is discoid LE (DLE). ${ }^{2}$ Signs and symptoms of CLE subtypes can include itch, scale, photosensitivity, erythema, alopecia, scar and fatigue. $^{3}$

There is a wide range of severity in CLE, and the skin lesions can be characterised as active or damage. The active lesions will appear scaly and erythematous, while the damage presents as dyspigmentation and scarring. CLE lesions can be chronic in nature, and according to Klein et al, patients with CLE experience a quality of life (QoL) that is similar to or worse than patients with chronic hypertension, congestive heart failure or diabetes mellitus type $2 .{ }^{4}$ Available 
treatment options have only moderate rates of success, with approximately $50 \%$ of patients with CLE improving with hydroxychloroquine, regarded as the first-line treatment for CLE. ${ }^{5}$ CLE refractory to antimalarials is often refractory to second-line treatment, which can cause side effects such as liver enzymes elevation, immunosuppression and peripheral neuropathy. ${ }^{6}$ Prior studies have evaluated CLE QoL through quantitative measuring tools, such as the Skindex-29, Interpersonal Processes of Care survey (IPC-29) and Dermatology Life Quality Index (DLQI), among others. ${ }^{7-9}$ Ogunsanya et al, using a focus group approach, were among the first to capture CLE patient-reported outcomes with regard to QoL. ${ }^{10}$ The present study conducted similar, open-ended interviews. However, these conversations were conducted oneon-one in clinic rooms to provide a sense of privacy and security. According to Kaplowitz, ${ }^{11}$ individual interviews are 18 times more likely to raise more sensitive information than focus groups. Additionally, our questions were open-ended without reference to any specific symptoms to avoid influencing patient responses. Other studies such as Arevalo-Bermudez et al had subjects with lower median Cutaneous Lupus Activity and Severity Index (CLASI) scores in both activity and damage. ${ }^{8}$

This study aimed to identify the subjective experiences of patients with CLE through semistructured, qualitative interviews. Six themes were identified during these patient interviews: Self-Consciousness, Physical Signs/Symptoms, Fear of Disease Progression, Functional Decline, Unwanted Attention and Emotional Symptoms. These themes are certainly interlinked, as self-consciousness and fear of progression result from the development of physical signs/symptoms. The most commonly reported co-occurrence of themes were Self-Consciousness and Physical Signs/Symptoms.

\section{METHODS}

\section{Sample and consent procedure}

The subjects in this study were adults $(\mathrm{n}=16$, age 19-86 years) with CLE, previously enrolled in the CLE database $(n=486)$ of patients from the autoimmune dermatology clinic of the University of Pennsylvania. The prospective longitudinal CLE database tracks disease progression of patients over time using physician assessment tools and patient QoL surveys. Inclusion criteria in both the substudy and broader database include patients who are English-speaking adults ( $\geq 18$ years old) who are diagnosed with CLE. Participants were selected as a convenience sample and recruited after their standard of care visits, but consideration was given to recruit a representative cohort from the CLE database in terms of sex and ethnicity. The proportions of sex and race were comparable with the main database, as seen in table 1 . The average interview length was $17 \mathrm{~min}$.

Sample size determination was based on when no novel themes were elicited from additional interviews with three more additional interviews past saturation
Table 1 Patient demographic and clinical characteristics

\begin{tabular}{|c|c|c|}
\hline & Study N (\%) & Database N (\%) \\
\hline Age (years) & $47.5(34.5-65.5)$ & \\
\hline \multicolumn{3}{|l|}{ Sex } \\
\hline Male & $3(18.75)$ & $92(18.8)$ \\
\hline Female & $13(81.25)$ & $394(80.5)$ \\
\hline \multicolumn{3}{|l|}{ Race } \\
\hline Caucasian & $8(50)$ & $298(60.9)$ \\
\hline African American & $7(43.75)$ & $162(33.1)$ \\
\hline $\begin{array}{l}\text { Asian/Pacific } \\
\text { Islander }\end{array}$ & $1(6.25)$ & 19 (3.9) \\
\hline
\end{tabular}

\begin{tabular}{|c|c|c|}
\hline \multicolumn{3}{|l|}{ Ethnicity } \\
\hline Hispanic & $0(0.0)$ & $22(4.5)$ \\
\hline Non-Hispanic & 16 (100) & 467 (95.5) \\
\hline \multicolumn{3}{|l|}{ CLE subtype } \\
\hline SCLE & $3(18.75)$ & \\
\hline DLE & $10(62.5)$ & \\
\hline
\end{tabular}

DLE +SCLE overlap 3 (18.75)

Systemic versus cutaneous

$\begin{array}{ll}\text { SLE +CLE } & 7(43.75) \\ \text { CLE } & 9(56.25)\end{array}$

Current disease activity (CLASI)

\begin{tabular}{|c|c|}
\hline Mild (0-9) & $7(43.5)$ \\
\hline Moderate (10-19) & $6(37.5)$ \\
\hline Severe $(\geq 20)$ & $2(12.5)$ \\
\hline Unknown & $1(6.25)$ \\
\hline \multicolumn{2}{|c|}{ Current disease damage (CLASI) } \\
\hline Mild (0-5) & 5 (31.25) \\
\hline Moderate (6-17) & $7(43.75)$ \\
\hline Severe $(\geq 18)$ & $3(18.75)$ \\
\hline Unknown & $1(6.25)$ \\
\hline \multicolumn{2}{|c|}{ Time since diagnosis (years) } \\
\hline Less than 1 year & $3(18.75)$ \\
\hline $1-5$ years & $2(12.5)$ \\
\hline $5-10$ years & $2(12.5)$ \\
\hline More than 10 years & $9(56.25)$ \\
\hline $\begin{array}{l}\text { Disease duration } \\
\text { (years, } 95 \% \mathrm{Cl} \text { ) }\end{array}$ & 14 (5.43 to 22.57$)$ \\
\hline
\end{tabular}

CLASI, Cutaneous Lupus Activity and Severity Index;

CLE, cutaneous lupus erythematosus; DLE, discoid lupus erythematosus; SCLE, subacute CLE.

for confirmation. Similar qualitative studies exploring patients' psychosocial concerns included comparable numbers of subject recruitment. ${ }^{10-14}$ The participants were presented with the opportunity to answer two questions verbally in addition to the surveys administered as part of the database visit. These database visits occur after their standard of care visits. Those subjects who expressed interest in participation were verbally consented to the 
substudy protocol by a research coordinator. Patients or the public were not involved in the design, or conduct, or reporting, or dissemination of this research. In order to preserve anonymity and privacy, subjects were assigned a study number (1-16) separate from their CLE database study number. Each transcript was de-identified.

\section{Data collection}

Interviews were conducted in person by a clinical research coordinator and were transcribed by another research coordinator in the room in order to capture the exact responses for reliable data analysis. Using scribes in qualitative research as an alternative to audio-recorded transcription has been shown to yield highly similar thematic analyses. ${ }^{15}$ Both research coordinators were trained in qualitative interviewing by an experienced qualitative researcher. Two open-ended questions were asked:

1. What troubles you most about your cutaneous/skin lupus in terms of the way it looks or feels? How much bother does the skin lupus cause?

2. What about your skin disease that most impacts your daily life?

The patients were allowed to elaborate in as much depth as they felt comfortable sharing, for as long as they felt comfortable sharing. Discussion was guided by a moderator guide which provided a script to ensure consistency. Current activity and damage of the skin lupus were evaluated by a physician in accordance with the CLASI tool after the interview for 15 of the 16 subjects. Clinical characteristics and demographics were collected prior to the interview with standardised forms used in the database.

\section{Data analysis}

The preliminary content analysis was conducted on a portion of transcripts $(12.5 \%)$ that was read multiple times and individually coded by both DZ and DY during the study. These preliminary codes were defined, compared and negotiated into a collaborative codebook by which the remainder of the transcripts was analysed. The initial inter-rater reliability of independent coders was $90 \%$. Differences in coders' perspectives were resolved through discussion. New codes were added to the codebook as novel concepts were mentioned by subjects. Data saturation was attained when subsequent interviews no longer elicited novel concepts. Three additional interviews were conducted past the point of saturation to ensure the maximum amount of relevant patient QoL information had been captured. DZ and DY sorted and combined the codes into a list of broader themes and subthemes. Each transcript was read twice more following the conclusion of data collection in order to confirm that the themes accurately and holistically reflected patient responses.

The frequency of certain themes was compared across age groups, sex, race, disease duration and CLE subtype (DLE vs SCLE). Responses were also correlated with the CLASI score of the patient. Themes reported $\geq 2$ times within a group were considered to be suggestive of a pattern.

\section{RESULTS}

A total of 16 patients were included in the study, with the sex and race composition comparable with the main CLE database study as seen in table 1 . The American College of Rheumatology criteria were used for the SLE diagnosis. CLE subtypes were determined by clinical diagnosis, and sex, race, ethnicity and current smoking status were selfreported by patients. CLASI score was calculated by an experienced physician during the interview.

Table 1 summarises the demographic and clinical features of the current study subjects. The sample was $81 \%$ female $(\mathrm{M}=3, \mathrm{~F}=13)$, had a median age of 47.5 years (19-86 years) and were almost evenly split between Caucasian (50\%) and African American (43.75\%) with one Asian American (6.25\%). All subjects were non-Hispanic and had CLE subtypes of SCLE (18.75\%), DLE (62.5\%) and DLE/SCLE overlap (18.75\%), with $43.75 \%$ having a concomitant diagnosis of SLE. The median duration of disease was 14 (5.43 to 22.57) years, and the median current activity and damage of disease, as determined by CLASI score at the time of the interview, were 10 (7.61 to 12.39 ) and 12 (8.51 to 15.49), respectively. Seven patients had mild CLE activity, as defined by a CLASI score of 0-9, six had moderate disease activity (CLASI score 10-19) and two had severe disease activity (CLASI score $\geq 20$ ). Regarding time since diagnosis of CLE, $18.75 \%$ were newly diagnosed ( $<1$ year), $25 \%$ were diagnosed between 1 and 10 years ago and $56.25 \%$ were diagnosed for over 10 years. Categorisation of the transcripts led to the establishment of six themes, each being composed of several subthemes (table 2).

The three most common themes were SelfConsciousness, Physical Signs/Symptoms and Fear of Disease Progression. The least prevalent themes $(n=8)$ were Unwanted Attention and Emotional Symptoms. The theme of Self-Consciousness reflects internalised insecurities that could be exacerbated by remarks made to the patient by other people. The theme includes comments about feeling different, the desire to cover up lesions and body image concerns. For example, one patient stated:

....as far as the way it looks, it's hideous. I can't go without a hat. I don't like wearing hats but I feel like I'm forced to. I feel embarrassed, ashamed of it. I wish I didn't have it.

The Physical Signs/Symptoms theme, the second most common, encompasses all comments made about the dermatological signs/symptoms directly discussed by the patient. For example:

[What bothers me is] more or less like the dry or itchiness...If I didn't have the white patches...or less like the rash...the pink or the red, I would be a little more at ease.

Fear of Disease Progression envelopes direct, primary statements of this fear, or secondary fears, such as significant anxiety regarding sun exposure or other CLE triggers. An example of each concern is: 


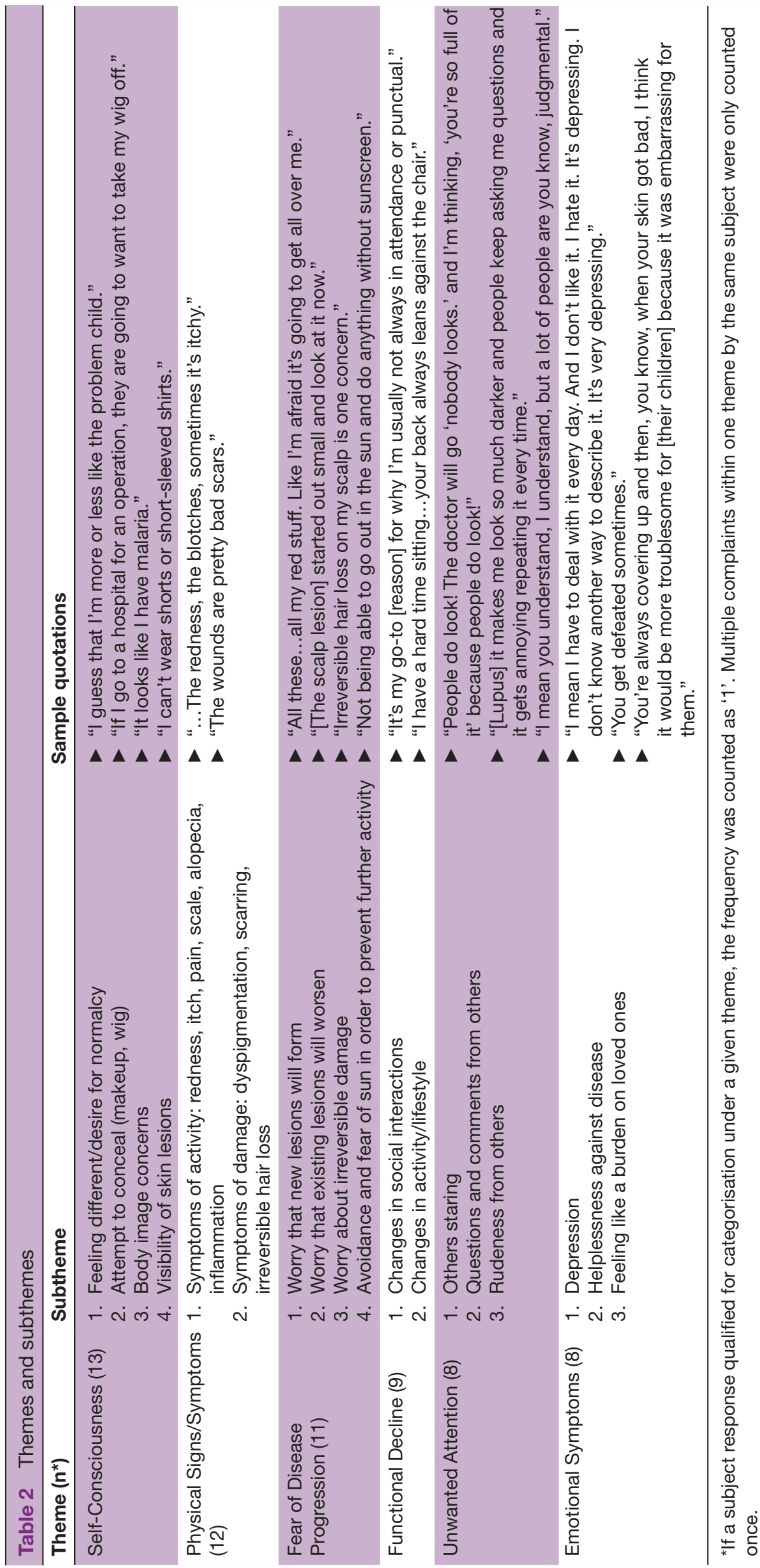


The only thing I'm concerned about is more hair loss...I mean the hair loss, one day I will wake up and I'm not going to have anything up there. That's a big deal. That's a big deal.

I would have to say you are always aware when the sun's out. Most people don't think about those things. I call the sun my kryptonite. It's generally what you think about $24 / 7$. The blinds are closed on a sunny day, I have to keep them closed... It's just literally your focal point...It's a tough way to live.

The theme of Functional Decline included any change to normal daily activity attributed to the patient's CLE. In addition to the common avoidance of the sun due to fear of disease exacerbation, subjects frequently described staying home or avoiding activity, especially during a flare.

"I have a hard time sitting. Your back always leans against the chair and I put the pillow up high...prevents me from sitting well." For instance, this patient with DLE had a large eroded area on their back which made it painful and difficult to sit.

Just basically when I get a flare, I don't like it. It's red, it's puffy, it's nasty looking, I stay in the house in the bed. If it's nothing major, I don't do it.

I guess you can say self-conscious about going out... other places like going out to eat at a restaurant, I'm more or less like let's takeout.

This finding echoes a pre-established pattern of isolation in patients with CLE and SLE. ${ }^{10}$ As a result of the interaction between functional decline and avoidance of unwanted attention, patients tend to stay at home more habitually.

As described above, Unwanted Attention encompassed experiences of negativity towards CLE coming from external sources, rather than stemming from the patient. This included staring, questions, comments or rudeness:

People would want to know, 'What's on your skin? Don't touch me. Oh my god, can I get that?'...You know, but, that was a problem going out with the skin and the stares and the looks. People fear what they don't know.

The last theme, Emotional Symptoms, meant to capture the health burden imposed by CLE in addition to the physical symptoms, such as depression, feeling like a burden and hopelessness, all of which were prominent concerns for patients with lupus. The specific mention of depression occurred in 4 of 16 interviews:

I mean I have to deal with it every day. And I don't like it. I hate it. It's depressing. I don't know another way to describe it. It's very depressing.

When you don't have light, you can get depressed. So, you have to trade off, do I want to get a rash or do I want to live with depression? Sometimes I have to go with the depression. And that's the hard part.
The commonly co-reported themes were SelfConsciousness and Physical Signs/Symptoms ( $\mathrm{n}=10$, $62.5 \%$ ), then followed by Fear of Disease Progression and Physical Signs/Symptoms, Fear of Disease Progression and Self-Consciousness, and Fear of Disease Progression and Unwanted Attention. The prior three overlaps were all found in half of the subjects $(n=8,50 \%)$.

\section{Relationship of themes and demographic factors Age}

Younger patients $(<60$ years old $)$ more frequently reported emotional symptoms. Younger patients (70\%) were much more likely than their older counterparts (16.7\%) to report emotional burden, including depression and hopelessness, as a result of their lupus. Additionally, $100 \%$ of older patients discussed physical symptoms as compared with $60 \%$ of younger patients. The number and type of mentioned physical symptoms, which ranged from one to six different symptoms, however, did not vary among age groups.

\section{Sex}

While there was a smaller cohort of men $(n=3)$ versus women $(n=16)$, the most striking finding is that all men mentioned physical symptoms, as opposed to $69 \%$ of women. Additionally, there was no variation in specific physical symptoms reported between sexes. Women more frequently mentioned Unwanted Attention (54\% vs $33 \%$ ), Self-Consciousness ( $85 \%$ vs $67 \%$ ), Emotional Symptoms (54\% vs $33 \%)$ and Functional Decline $(62 \%$ vs $33 \%$ ).

\section{Race}

The most common themes mentioned by Caucasian subjects $(n=8)$ were Fear of Disease Progression $(75 \%)$ and Physical Signs/Symptoms (75\%). The most common themes mentioned by African American subjects $(n=7)$ were Self-Consciousness (100\%) and Physical Signs/ Symptoms (71\%).

Of the subjects reporting Physical Signs/Symptoms, African Americans were more likely to report alopecia and dyspigmentation. Perhaps relatedly, all $(\mathrm{n}=7)$ African American participants mentioned Self-Consciousness, whereas five out of eight Caucasian participants discussed this theme.

\section{Current smoking status}

Fear of Disease Progression (80\% vs 64\%) and Emotional Symptoms ( $80 \%$ vs $36 \%$ ) were more frequently mentioned by current smokers $(n=5)$ compared with non-smokers $(\mathrm{n}=11)$.

\section{Physical symptoms}

There was a wide variation in type of physical signs/symptoms reported (figure 1). The commonly discussed physical symptoms included erythema, dyspigmentation, scar and alopecia. Pain and itch were also frequently brought up. 


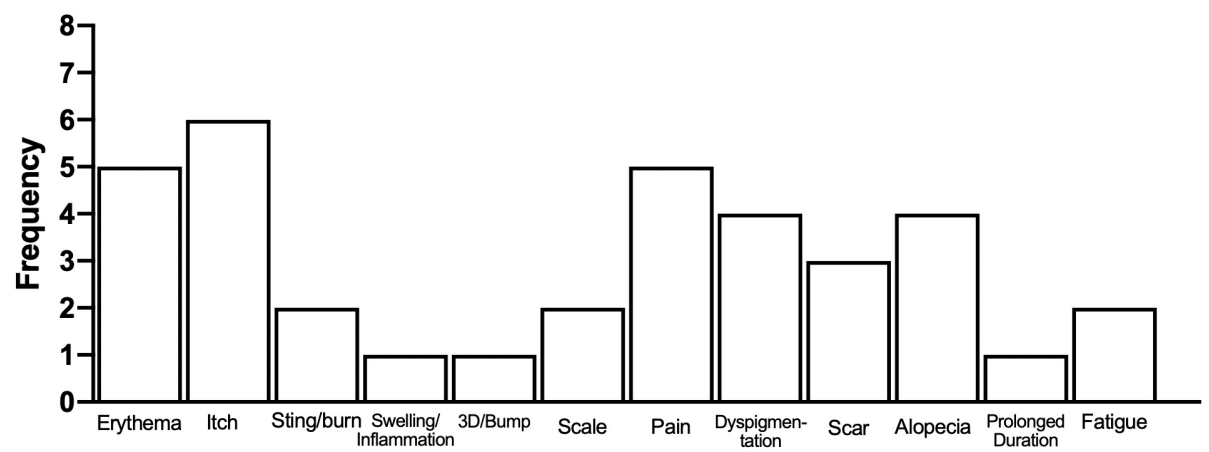

Figure 1 Frequency of reported physical signs/symptoms. If a subject response qualified for categorisation under a given symptom label, the frequency was counted as ' 1 '. Multiple complaints regarding the same sign/symptom by the same subject were only counted once.

Erythema and scale, among other signs, are grouped as 'Activity' symptoms and dyspigmentation and scar are grouped under 'Damage'. Alopecia can be qualified as activity or damage depending on if it is ongoing or a result of a scarred scalp. All patients with a CLASI activity score at or above $15(\mathrm{n}=3)$ mentioned Emotional Symptoms during their interview. For those with CLASI activity scores of 8 or higher, 6 of 11 subjects reported Emotional Symptoms.

\section{Diagnosis and duration}

A significant variation in reported themes among subjects with DLE versus subjects with SCLE was not noted, although all $(n=10)$ patients with DLE discussed SelfConsciousness, whereas this theme was only discussed by $66.7 \%$ of subjects with SCLE $(n=3)$. Notably, all subjects with multiple CLE subtypes $(n=3)$ mentioned Fear of Disease Progression and Physical Signs/Symptoms. The type of physical signs/symptoms did not vary between diagnostic groups. When comparing subjects with CLE versus CLE/SLE, those with SLE experienced more unwanted attention and more fear of disease progression. Similarly, subjects on more medications, and thus presumably experiencing more severe disease, tended to report more physical signs/symptoms.

However, although diagnosis (CLE vs CLE/SLE) impacted the rate of theme occurrence, duration of disease did not. Those with a longer history of disease were no more or less likely to report activity, damage or overall physical symptoms than their newly diagnosed counterparts. Subjects who had been diagnosed $<1$ year were equally likely to report Emotional Symptoms (66.7\%) compared with those who had been diagnosed $>10$ years ago $(66.7 \%)$.

\section{DISCUSSION}

Previous QoL instruments including the DLQI, Skindex29, Short Form 36 and Visual Analogue Scales were not developed specifically for patients with CLE, and therefore do not completely portray the experience of these patients. ${ }^{10}$ Qualitative research can be a powerful tool that offers a deeper insight into the psychosocial concerns of patients compared with standardised QoL surveys. Thus, there is a need to identify and elaborate on concerns unique to patients with CLE.

However, there is a paucity of qualitative research regarding CLE QoL. Thematic synthesis of 46 qualitative studies conducted by Sutanto et al shows that patients with SLE experience themes of societal stigma, self-consciousness and prognostic uncertainty. ${ }^{16}$ Selfconsciousness in SLE can be driven by patients' perspectives of other people's perspectives of their bodies. ${ }^{17}$ Ogunsanya et al used focus groups to probe how CLE affects QoL and discovered themes are similar to our findings. ${ }^{10}$ Their findings show that patients with CLE cited concerns such as cutaneous manifestations, social anxiety and fear of progression. However, they did not parse for recurrent themes among patient characteristics, such as sex or race, nor was the lupus activity captured. Our study identified six themes: Self-Consciousness, Physical Signs/Symptoms, Fear of Disease Progression, Functional Decline, Unwanted Attention and Emotional Symptoms.

The frequency of these themes was calculated according to patient characteristics. For instance, all men mentioned Physical Signs/Symptoms, while women more frequently mentioned Unwanted Attention (54\% vs 33\%), SelfConsciousness ( $85 \%$ vs $67 \%$ ), Emotional Symptoms ( $54 \%$ vs 33\%) and Functional Decline (62\% vs 33\%). Generally, women express more body image dissatisfaction and worse QoL, specifically in the CLE population. ${ }^{418}$

The most common theme mentioned by African American subjects $(n=7)$ was Self-Consciousness $(100 \%)$. Of the subjects reporting Physical Signs/Symptoms, African Americans were more likely to report alopecia and dyspigmentation. In regard to complaints of alopecia, it is interesting to note that all African American subjects with CLE brought up hair loss. DLE lesions, which favour the scalp and often cause scarring, are more common among African Americans in our database population, as well as globally, and could help explain this finding. ${ }^{19}$

This study had 5 smokers and 11 non-smokers. Smoking is known to exacerbate many dermatological conditions, such as hidradenitis suppurativa and psoriasis. ${ }^{20}{ }^{21}$ Arevalo-Bermudez et al's CLE cohort found that 
nicotine dependence can confer up to 10 times worse QoL, even after controlling for other variables. ${ }^{22}$ These themes can offer insight to the high rates of depression that can affect up to $30 \%$ of individuals with CLE. ${ }^{9}$ The finding of significant differences in the disclosure of psychological, mental or emotional strain between age groups supports Klein et al's previous findings regarding younger age and emotional impairment. ${ }^{4}$ However, Ishiguro et al's study of Japanese patients with CLE identified older age as a risk factor for poorer QoL. ${ }^{23}$ Patients with CLE over 60 years of age reported emotional symptoms less frequently (16.7\%) compared with patients under 60 years $(70 \%)$, while there was no pattern of increased emotional symptoms with increased disease duration. This suggests that increasing age has a greater role in frequency of emotional symptoms compared with disease duration. Gupta and Gupta's work on the QoL of patients with psoriasis found similar results in regard to increased age and lessened psychosocial impact of cutaneous disease. ${ }^{24}$ Contributing factors to this discrepancy include better establishment of a social support system and lessening importance of social stigma related to age, as well as presumably more financial security. ${ }^{24}$

There was no difference in frequency of reports falling under a given theme between CLE subtypes, that is, between patients with DLE versus SCLE. Patients with CLE with SLE overlap did however experience more unwanted attention and fear of disease progression. The latter is logical, as a diagnosis of systemic lupus involves greater lifetime risk of morbidity and mortality. Prior QoL studies of patients with SLE specifically have concluded that patients with SLE frequently either feel or fear that those around them will not accept their condition, and that there is a lack of understanding from loved ones. ${ }^{25}$ This potentially explains the coexistence of unwanted attention and SLE status, in that perhaps patients with CLE/SLE overlap experience more debilitating disease, and as a result, more questions and negative remarks from friends and family.

The demographics of this study were representative of CLE incidence in the population seen by the Autoimmune Dermatology group at the University of Pennsylvania. However, as with any qualitative interview study, in which saturation is often reached at small sample sizes, the results may not be generalisable to the CLE population as a whole. Generalisability can also be affected by sample collection method. This study also used a convenience sample rather than a purposive sample. Purposive sampling has greater efficacy compared with convenience sampling ${ }^{26}$ and the additional themes that may have been drawn out through the information-rich individuals of a purposive sample. It stands to reason, additionally, that the patients seen at the University of Pennsylvania may not experience or disclose all possible CLE-related QoL themes, and therefore continued interview-based work with these patients using a purposive sample is necessary. In the future, looking at more specific patient characteristics such as comorbidities and comparing them with this original study would be interesting.
Due to the small sample size, further work also needed to elucidate response patterns according to patient characteristics that are known to impact QoL, such as other comorbidities that are known to decrease QoL. While the use of a convenience sample allowed for a wide variety of patient perspectives, specific pattern analyses based on other disease factors will require further investigation. For instance, no patients brought up concerns about sexual issues or relations within the themes of Self-Consciousness or Functional Decline, although they are included in skin-specific QoL surveys, such as the Skindex-29 or the DLQI. The data and subsequent conclusions were not shared with patients with CLE outside of this study, which limits cross-verification of themes.

This study captures concerns of patients with CLE and raises awareness of how different groups of patients with CLE are impacted. These themes include psychological and physical distresses, impaired functionality and dysfunctional social interactions that arise from CLE. The patient experiences and resultant themes elucidated by this study are worth noting in future standardised estimations of the QoL of patients with CLE. Specifically, young age and female sex are two important risk factors for psychological distress in CLE, and providers should discuss these topics when evaluating patient disease progression.

Contributors DZ, DY, SC, RK, JC and SA contributed to conception and design of study, data collection and data analysis. RF contributed to data analysis and interpretation. J0 obtained funding and contributed to the conception of study. VPW obtained funding and supervised the study. All authors were involved in manuscript preparation and review, and approved the final version for publication.

Funding Supported by the National Institutes of Health (R01AR071653), the Lupus Foundation of America, Philadelphia Tri-State Chapter and the US Department of Veterans Affairs (Veterans Health Administration, Office of Research and Development and Biomedical Laboratory Research and Development).

Competing interests VPW has been a consultant for Medlmmune, AstraZeneca, Pfizer, Biogen, Celgene, Resolve, Janssen, Gilead, Lilly, BMS, Nektar, Abbvie, Akira, Viela, GSK, EMD Serona and has received a grant from Celgene, Amgen, Janssen, Biogen, Gilead, Viela and AstraZeneca. The University of Pennsylvania owns copyright over the CLASI.

Patient and public involvement Patients and/or the public were not involved in the design, or conduct, or reporting, or dissemination plans of this research. Patient consent for publication Not required.

Ethics approval This study was approved by the Institutional Review Board at the University of Pennsylvania (\#805758).

Provenance and peer review Not commissioned; externally peer reviewed. Data availability statement Data are available upon reasonable request.

Open access This is an open access article distributed in accordance with the Creative Commons Attribution Non Commercial (CC BY-NC 4.0) license, which permits others to distribute, remix, adapt, build upon this work non-commercially, and license their derivative works on different terms, provided the original work is properly cited, appropriate credit is given, any changes made indicated, and the use is non-commercial. See: http://creativecommons.org/licenses/by-nc/4.0/.

ORCID iD

Daisy Yan http://orcid.org/0000-0003-0863-7201

\section{REFERENCES}

1 Bolognia J, Schaffer JV, Cerroni L. Dermatology. Fourth edition, 2021.

2 Okon LG, Werth VP. Cutaneous lupus erythematosus: diagnosis and treatment. Best Pract Res Clin Rheumatol 2013;27:391-404. 
3 Patel P, Werth V. Cutaneous lupus erythematosus: a review. Dermato Clin 2002;20:373-85.

4 Klein R, Moghadam-Kia S, Taylor L, et al. Quality of life in cutaneous lupus erythematosus. J Am Acad Dermatol 2011;64:849-58.

5 Kuhn A, Ruland V, Bonsmann G. Cutaneous lupus erythematosus: update of therapeutic options Part II. J Am Acad Dermatol 2011;65:e195-213.

6 Callen JP, Jorizzo JL, Bolognia JL. Dermatological signs of internal disease E-Book: expert Consult-Online and print: Elsevier health sciences, 2009.

7 Ogunsanya ME, Cho SK, Hudson A, et al. Validation and reliability of a disease-specific quality-of-life measure in patients with cutaneous lupus erythematosus. Br J Dermatol 2019;180:1430-7.

8 Arévalo-Bermúdez MP, Pita-Fernández S, Paradela S, et al. Cutaneous lupus erythematosus: quality of life and related factors in a cohort of 260 patients from a Coruña, Spain. Br J Dermatol 2018;178:e117-8.

9 Hong J, Aspey L, Bao G, et al. Chronic cutaneous lupus erythematosus: depression burden and associated factors. Am J Clin Dermatol 2019;20:465-75.

10 Ogunsanya ME, Brown CM, Lin D, et al. Understanding the disease burden and unmet needs among patients with cutaneous lupus erythematosus: a qualitative study. Int $J$ Womens Dermatol 2018;4:152-8.

11 Kaplowitz MD. Statistical analysis of sensitive topics in group and individual interviews. Quality and Quantity 2000;34:419-31.

12 van Scheppingen C, Lettinga AT, Duipmans JC, et al. Main problems experienced by children with epidermolysis bullosa: a qualitative study with semi-structured interviews. Acta Derm Venereol 2008;88:143-50.

13 Esmann S, Jemec GBE. Psychosocial impact of hidradenitis suppurativa: a qualitative study. Acta Derm Venereol 2011;91:328-32.

14 Weiss M, Weisshaar E. Qualitative interviews on chronic pruritus in haemodialysis patients. Acta Derm Venereol 2014;94:713-4.
15 Eaton K, Stritzke WG, Ohan JL. Using scribes in qualitative research as an alternative to transcription. The Qualitative Report 2019;24:586-605.

16 Sutanto B, Singh-Grewal D, McNeil HP, et al. Experiences and perspectives of adults living with systemic lupus erythematosus: thematic synthesis of qualitative studies. Arthritis Care Res 2013:65:1752-65.

17 Hale ED, Treharne GJ, Lyons AC, et al. "Joining the dots" for patients with systemic lupus erythematosus: personal perspectives of health care from a qualitative study. Ann Rheum Dis 2006;65:585-9.

18 MacNeill LP, Best LA, Davis LL. The role of personality in body image dissatisfaction and disordered eating: discrepancies between men and women. J Eat Disord 2017;5:44.

19 Hordinsky M. Cicatricial alopecia: discoid lupus erythematosus. Dermatol Ther 2008;21:245-8.

20 Pezzolo E, Naldi L. The relationship between smoking, psoriasis and psoriatic arthritis. Expert Rev Clin Immunol 2019;15:41-8.

21 Bukvić Mokos Z, Miše J, Balić A, et al. Understanding the relationship between smoking and hidradenitis suppurativa. Acta Dermatovenerol Croat 2020;28:9-13

22 Arévalo-Bermúdez MP, Pita-Fernández S, Paradela S, et al. Cutaneous lupus erythematosus: quality of life and related factors in a cohort of 260 patients from a Coruña, Spain. Br J Dermatol 2018;178:e117-8.

23 Ishiguro $\mathrm{M}$, Hashizume $\mathrm{H}$, Ikeda $\mathrm{T}$, et al. Evaluation of the quality of life of lupus erythematosus patients with cutaneous lesions in Japan. Lupus 2014;23:93-101.

24 Gupta MA, Gupta AK. Age and gender differences in the impact of psoriasis on quality of life. Int J Dermatol 1995;34:700-3.

25 Olesińska M, Saletra A. Quality of life in systemic lupus erythematosus and its measurement. Reumatologia 2018;56:45-54.

26 Vasileiou K, Barnett J, Thorpe S, et al. Characterising and justifying sample size sufficiency in Interview-Based studies: systematic analysis of qualitative health research over a 15-year period. BMC Med Res Methodol 2018;18:148. 